\title{
UNA APLICACION DE LOS DISEÑOS DE RESPUESTA ALEATORIZADA AL CONSUMO DE CANNABIS
}

Jesús BASULTO SANTOS

Carlos ARIAS MARTIN

\section{INTRODUCCION}

El objetivo del presente trabajo es, en primer lugar, describir algunos diseño de respuesta aleatorizada, en donde se han obviado todos los planteamiento matemáticos, y en segundo lugar, presentar los resultados obtenidos en la encues ta realizada a los alumnos de la Facultad de Ciencias Económicas y Empresaria les de la Universidad de Sevilla.

Con esta investigación empírica se pretendían cubrir dos objetivos fundamen tales; en primer lugar, valorar la actitud de los entrevistados cuando se les realiz; este tipo de encuestas, y en segundo lugar, aplicar los resultados teóricos qu estábamos obteniendo.

De cualquier forma, el lector interesado en la formulación y desarrollo de lo: diferentes diseños, puede acudir a la tesis doctoral "Análisis Bayesiano de lo: Diseños de Respuesta Aleatorizada" de Arias, C. cuya Iectura y defensa se reali zó en la Facultad de Ciencias Económicas y Empresariales de la Universidad d Sevilla en diciembre de 1990.

\section{DISEÑOS DE RESPUESTA ALEATORIZADA}

Cuando se realiza una encuesta por muestreo se pueden cometer dos tipos $d \epsilon$ errores; los denominados errores debidos al muestreo y los errores ajenos a muestreo. Nuestro trabajo se va a centrar en los errores ajenos al muestreo, y más concretamente, en aquellos debidos a las propias características de los individuos que se pretenden analizar, que bien porque no estén consideradas socialmente 
deseables, bien porque sean altamente personales, o bien porque sean ilegales, tienen la consideración de íntimas.

Así, cuando se pretende estimar la proporción de la población que, por ejemplo, consume cierto tipo de drogas, no es adecuado preguntar directamente a un individuo dado si posee o no esta característica íntima, puesto que tanto los porcentajes correspondientes a la no respuesta, como los debidos a contestaciones deliberadamente falsas así lo aconsejan. Para resolver estas situaciones surgen los diseños de respuesta aleatorizada, en los que a cada uno de los entrevistados elige, mediante un mecanismo de aleatorización que se le muestra, una de entre dos o más preguntas que le son presentadas, sin que el entrevistador conozca el resultado de dicha selección.

Esta técnica fue concebida por Warner (1965), y su diseño supone que la población queda dividida en dos grupos no solapados, cuya unión es el colectivo objeto de estudio, donde el primer grupo lo constituyen aquellos individuos que poseen la característica íntima, mientras que el segundo lo forman los que no la tienen. $\mathrm{Y}$ así, construye dos preguntas o declaraciones relacionadas, esto es;

Pertenezco al primer grupo.

Pertenezco al segundo grupo.

haciendo declarar a cada individuo seleccionado si pertenece o no al grupo que le haya correspondido después de utilizar el mecanismo aleatorio. Este diseño fue generalizado posteriormente, a partir del supuesto de que los elementos de la población, en relación con la característica íntima, se pueden dividir en $\mathrm{k}$ niveles exhaustivos y mutuamente excluyentes, donde al menos 1 o a lo sumo $\mathrm{k}-1$, tienen la consideración de íntimo, por Abul-Ela et al (1967) y por Baurke y Dalenius (1973).

En la línea de proteger en mayor medida la intimidad de los entrevistados, aparece en 1967 el diseño con una pregunta no relacionada, propuesto por Horvitz et al y desarrollado notablemente por Greenberg et al. (1969). Este diseño crea una mayor incertidumbre al establecer dos preguntas o declaraciones, una íntima y otra intrascendente, donde esta última es independiente o está no relacionada con la primera, de forma que cada entrevistado debe responder si posee $o$ no la característica que le haya correspondido al utilizar el mecanismo aleatorio. Ahora bien, el diseño es distinto según la proporción de la población que posee la característica no relacionada sea conocida o bien tenga que estimarse mediante la encuesta.

El desarrollo de este segundo supuesto ha tenido más interés, puesto que aunque la característica intrascendente es seleccionada por el analista, no debe- 
mos perder de vista que el objetivo básico de los diseños de respuesta, aleatoriza da es proteger la intimidad de los entrevistados, y así, debe de ser imposibl verificar, para un individuo dado, si posee o no la característica no relacionada Para la utilización de este diseño, desde el punto de vista clásico, es necesari seleccionar dos muestras aleatorias simples con reemplazamiento, independiente y no solapadas, aunque en algunos supuestos, como el de Moors (1971), en uni de las muestras no se utilice mecanismo aleatorio alguno, dado que sólo st pregunta de forma directa si posee o no la característica intrascendente.

Este diseño, ha sido generalizado, con idénticos planteamientos que el ds Warner, por Greenberg et al (1969), Eriksson (1973) y Bourke (1974), extendién dose también al supuesto de dos preguntas no relacionadas en Folsom et al (1973).

Una característica fundamental de cualquier diseño de respuesta aleatorizada frente al que emplea la pregunta directa, es que su utilización conduce, bajo e supuesto de tamaños muestrales idénticos, a una disminución de la precisión de estimador, medida en términos de su desviación estándar.

Un camino para lograr incrementar la precisión del estimador sin aumentar e tamaño muestral es hacer uso de la inferencia bayesiana. Así, a partir de lé información inicial sobre la proporción de individuos que poseen la característič́ íntima, medida en términos de una distribución de probabilidad a priori o inicial podemos ofrecer estimaciones por intervalo, más precisas que las ofrecidas por la inferencia clásica, siendo el trabajo de Winkler y Franklin (1979), el primero que aborda desde esta concepción el diseño propuesto por Warner.

Por otra parte, la función de verosimilitud resultante al aplicar estos diseños es complicada, haciendo el cálculo del estimador máximo verosímil difícil de obtener. Una forma de resolver este problema es utilizar el algoritmo EM (alternancia entre tomar esperanza y maximizar), que aunque debido a Hartley (1958), nc aparece desarrollado adecuadamente hasta 1977 por Dempster et al. Así, en aquellas situaciones que producen datos no observados, se formaliza una idea intuitiva; esto es, reemplazar los valores no observados por valores estimados, estimar el parámetro, reestimar los valores no observados asumiendo que el valor estimado del parámetro es correcto, reestimar el parámetro, y así sucesivamente hasta conseguir la convergencia. La aplicación de este algoritmo a los diseños de respuesta aleatorizada, desde el punto de vista clásico, ha sido introducida por Bourke y Moran (1988).

Ahora bien, dado que para poder realizar inferencias no es suficiente aproximar la desviación estándar de la distribución a posteriori, se han utilizado diversos métodos para aproximar esta desviación estándar, que dan lugar a un conjunto de aproximaciones a la verdadera distribución final de la proporción de indivi- 
duos que poseen la característica íntima, utilizando los métodos propuestos por Leonard (1975), Laird y Louis (1982) y Tierney y Kadane (1986).

\section{CARACTERISTICAS DE LA ENCUESTA}

El colectivo objeto de estudio estaba formado por los alumnos de la Facultad de Ciencias Económicas y Empresariales de la Universidad de Sevilla, y el cuestionario, que se ofrece en el anexo, constaba de diez preguntas, donde dos atendían a variables de clasificación, otras dos se referían a la característica íntima que seleccionamos, mientras que con el resto de preguntas intentamos valorar la actitud de los entrevistados hacia este tipo de encuestas.

Para la elección de la característica íntima seguimos el trabajo "Los andaluces ante las drogas" (Junta de Andalucía, 1989), y así, se propuso preguntar si había consumido alguna vez, durante los últimos treinta días, Cánnabis (Porros); esto es, Haschis, Marihuana,... También, y siguiendo con el objetivo de la experimentación, a cada individuo, se le preguntaba, tanto según el diseño de Warner, como el de preguntas no relacionadas. Ahora bien, otro problema que había que resolver era la selección de la característica no relacionada. Así, se intentó aprovechar la información que suministraba el "Informe sobre actitudes, opiniones e imagen de la Universidad de Sevilla" (Diez de Castro et al, 1987), pero bien porque las variables consideradas podían tener a priori relación con el consumo de Cánnabis, bien porque podíamos conocer si el individuo poseía la característica no relacionada, tuvimos que acudir a una pregunta no considerada en el informe, y que al final fue si venía habitualmente en moto a la Facultad.

También, y siguiendo con el objetivo de la experimentación, se seleccionaron dos muestras del mismo tamaño, siendo la diferencia entre ellas la probabilidad con el que el instrumento aleatorio seleccionaba la pregunta íntima en el diseño de preguntas no relacionadas. Por lo que respecta al instrumento aleatorio, decidimos utilizar los naipes, porque pensamos que eligiendo uno que fuera conocido por los entrevistados, facilitábamos su comprensión hacia el método, lo que, en principio, nos conduciría a una mayor cooperación.

Así pues, cada individuo selecciona, en primer lugar, mediante el instrumento de aleatorización una de estas dos preguntas:

¿Pertenece al grupo de los consumidores?

¿Pertenece al grupo de los no consumidores? 
respondiendo simplemente SI o NO a la pregunta que le haya comespondid mientras que al aplicar el diseño de preguntas no relacionada, selecciona nuev: mente mediante el mecanismo de aleatorización una de estas dos preguntas:

¿Pertenece al grupo de los consumidores?

¿Viene en moto habitualmente a la Facultad?

respondiendo nuevamente SI o NO a la pregunta seleccionada, donde en cua quier caso, se considera consumidor todo aquél que haya consumido alguna ve: durante los últimos treinta días, Cánnabis.

\section{CARACTERISTICAS DE LA MUESTRA}

Dado que el número de alumnos matriculados en la Facultad, en el curs académico 1989-90 ascendía a 4.290, y puesto que lo que se pretende estimar e una proporción, aplicamos la fórmula habitual para determinar el tamaño mues tra, ascendiendo éste a 366. En función de la información disponible, estratifica mos la población por el curso superior en que se encontraba matriculado, reali zando la afijación de forma proporcional al tamaño de cada estrato. Las encuesta se realizaron desde el día 26 de marzo al 4 de abril de 1990.

El número de encuestas realizadas ascendió a 275 (75,13\%), siendo la distri bución por muestra:

Muestra 1: $137(74,86 \%)$

Muestra 2: $138(75,41 \%)$

Las características de las muestras, según las variables de clasificación, sol las que aparecen en las tablas I y II:

TABLA I

\begin{tabular}{lcccccc}
\hline $\begin{array}{l}\text { Edad } \\
\text { (años) }\end{array}$ & \multicolumn{2}{c}{ Total } & \multicolumn{2}{c}{ Muestra 1 } & \multicolumn{2}{c}{ Muestra 2 } \\
& $\mathrm{N}^{*}$ & $\%$ & $\mathrm{~N}^{\text {\% }}$ & $\%$ & $\mathrm{~N}^{\circ}$ & $\%$ \\
\hline $17-18$ & 50 & 18,19 & 21 & 15,33 & 29 & 21,01 \\
19 & 55 & 20,00 & 30 & 21,90 & 25 & 18,13 \\
20 & 53 & 19,27 & 27 & 19,71 & 26 & 18,84 \\
$21-22$ & 64 & 23,27 & 35 & 25,55 & 29 & 21,01 \\
$>22$ & 53 & 19,27 & 24 & 17,51 & 29 & 21,01 \\
\hline Total & 275 & 100,00 & 137 & 100,00 & 138 & 100,00 \\
\hline
\end{tabular}


TABLA II

\begin{tabular}{lcccccc}
\hline Sexo & \multicolumn{2}{c}{ Total } & \multicolumn{2}{c}{ Muestra 1 } & \multicolumn{2}{c}{ Muestra 2 } \\
& N $^{*}$ & $\%$ & No & $\%$ & No $^{\circ}$ & $\%$ \\
\hline Mujeres & 104 & 37,82 & 53 & 38,69 & 51 & 36,96 \\
Hombres & 171 & 62,18 & 84 & 61,31 & 87 & 63,04 \\
\hline Total & 275 & 100,00 & 137 & 100,00 & 138 & 100,00 \\
\hline
\end{tabular}

De las dos tablas anteriores podemos concluir que no se aprecian diferencias notables entre las dos muestras, en lo que se refiere a estas dos variables de clasificación.

\section{VALORACION DE LOS ENCUESTADOS SOBRE LOS DISEÑOS PROPUESTOS}

Después de que el alumno hubiera respondido SI o NO a la pregunta seleccionada según el diseño de Warner, se le preguntaba si creía que su intimidad quedaba adecuadamente protegida al utilizar la baraja en lugar de preguntarle directamente. A continuación, y después de cambiarle la baraja, debía responder nuevamente SI o NO a la pregunta impresa en la carta seleccionada por él, según el diseño de preguntas no relacionadas, y nuevamente se le hacía la misma pregunta sobre la protección de su intimidad. Los resultados obtenidos son los que se muestran en las tablas III y IV.

Así, podemos observar que el $87 \%$ de los encuestados considera que su intimidad queda adecuadamente protegida, sin diferencias notables según el diseño aplicado, aunque este porcentaje es ligeramente superior, para ambos diseños, en la muestra 1 .

TABLA III

\begin{tabular}{lrrrrrr}
\hline Warner & \multicolumn{2}{c}{ Total } & \multicolumn{2}{c}{ Muestra 1 } & \multicolumn{2}{c}{ Muestra 2 } \\
& № & $\%$ & № & $\%$ & \multicolumn{1}{c}{ No $^{2}$} & $\%$ \\
\hline \multirow{2}{*}{ SI } & 240 & 87,27 & 123 & 89,78 & 117 & 84,78 \\
NO & 35 & 12,73 & 14 & 10,22 & 21 & 15,22 \\
\hline \multirow{2}{*}{ Total } & 275 & \multirow{2}{*}{100,00} & 137 & 100,00 & 138 & 100,00 \\
\hline
\end{tabular}


TABLA IV

\begin{tabular}{lrrrrrrr}
\hline No relacionadas & \multicolumn{2}{c}{ Total } & \multicolumn{2}{c}{ Muestra 1 } & \multicolumn{2}{c}{ Muestra 2 } \\
& No & $\%$ & No & $\%$ & \multicolumn{2}{c}{$N^{2}$} & $\%$ \\
\hline \multirow{2}{*}{ SI } & 238 & 86,54 & 124 & 90,51 & 114 & 82,61 \\
NO & 37 & 13,46 & 13 & 9,49 & 24 & 17,39 \\
\hline \multirow{2}{*}{ Total } & 275 & 100,00 & 137 & $\mathbf{1 0 0 , 0 0}$ & 138 & 100,00 \\
\hline
\end{tabular}

También, se pidió que valorasen cual de los dos diseños propuestos protegí más su intimidad. De esta forma, y teniendo en cuenta sus respuestas sobre cad. uno de ellos por separado, podemos ofrecer la tabla $\mathrm{V}$, donde se observa que par: el 58\% de los entrevistados no existe diferencia entre ambos diseños, consideran do que ambos protegen de igual forma su intimidad, mientras que existe u porcentaje algo superior de alumnos que piensan que es mejor el diseño dı preguntas no relacionadas que el de Warner; que alrededor del $9 \%$ considera qu ninguno de los dos diseños es suficiente para que su intimidad quede protegida, ? que únicamente hay tres individuos que a pesar de haber manifestado que ningu no de los diseños propuestos protege adecuadamente su intimidad, se inclinan : valorar uno superior a otro.

\section{TABLA V}

\begin{tabular}{lrrrrrr}
\hline & \multicolumn{2}{c}{ Warner } & & \\
\cline { 2 - 6 } Diseño & \multicolumn{2}{c}{$\begin{array}{c}\text { SI } \\
\text { No relac. }\end{array}$} & \multicolumn{2}{c}{ NO No relac. } & \multicolumn{2}{c}{ Total } \\
& SI & NO & SI & NO & No & $\%$ \\
\hline Warner & 33 & 7 & 0 & 2 & 42 & 15,27 \\
No relac. & 38 & 0 & 9 & 1 & 48 & 17,45 \\
Ninguno & 0 & 0 & 0 & 25 & 25 & 9,10 \\
Por igual & 160 & 0 & 0 & 0 & 160 & 58,18 \\
\hline Total & 231 & 7 & 9 & 28 & 275 & 100,00 \\
\hline
\end{tabular}

Además, hemos pretendido que los entrevistados indicaran cuál de los diseño: propuestos les parecía más claro. En este sentido ofrecemos la tabla VI.

Si bien los diseños con preguntas no relacionadas surgieron, fundamentalmen. te, para obtener una actitud más favorable por parte de los entrevistados, puestc que crea una incertidumbre sobre si se está respondiendo a la pregunta íntima o : la no relacionada, podemos comprobar que este hecho no queda debidament 
TABLA VI

\begin{tabular}{|c|c|c|c|c|c|c|}
\hline \multirow{2}{*}{$\begin{array}{l}\text { Diseño } \\
\text { Claridad }\end{array}$} & \multicolumn{2}{|c|}{ Total } & \multicolumn{2}{|c|}{ Muestra 1} & \multicolumn{2}{|c|}{ Muestra 2} \\
\hline & $\mathrm{N}^{\mathrm{a}}$ & $\%$ & $\mathrm{~N}^{\mathrm{e}}$ & $\%$ & № & $\%$ \\
\hline Warner & 125 & 45,45 & 57 & 41,61 & 68 & 49,28 \\
\hline No relac, & 19 & 6,91 & 14 & 10,22 & 5 & 3,62 \\
\hline Ninguno & 60 & 21,82 & 31 & 22,63 & 29 & 21,01 \\
\hline Por igual & 71 & 25,82 & 35 & 25,54 & 36 & 26,09 \\
\hline Total & 275 & 100,00 & 137 & 100,00 & 138 & 100,00 \\
\hline
\end{tabular}

reflejado en la tabla $\mathrm{V}$, puesto que únicamente el $17 \%$ de los entrevistados se manifiestan en este sentido. Ahora bien, nosotros pensábamos que el diseño de Warner podía crear cierta confusión en la pregunta complementaria; o sea, en si pertenece al grupo de los no consumidores. Sin embargo, el $45 \%$ de los alumnos eligen como diseño más claro el de Warner, frente al 7\% que se inclinan por el de preguntas no relacionadas. Creemos que estas situaciones se deben al hecho de los entrevistados no comprenden que tiene que ver el ser consumidor o no, con venir habitualmente en moto a la facultad o no. Y precisamente esta falta de claridad en la comprensión del diseño, que no de las preguntas, es lo que ha motivado los resultados expuestos en la tabla $\mathrm{V}$ y sobre todo en la VI.

También, debemos resaltar que el $22 \%$ de los entrevistados no considera que ninguno de los diseños sea claro, y ello a pesar de que a cada entrevistado se le iba mostrando la composición de las barajas, indicándole que fuera el mismo el que barajara los naipes, que extrajera uno, que sólo debía ser visto por él, y que después de contestar SI o NO, volviera a introducir el naipe en la baraja, moviendo de nuevo las cartas para que nadie pudiera saber la pregunta que le había correspondido. Nuevamente nos inclinamos a pensar que este resultado se debe, en gran medida, en la falta de comprensión del diseño, que no de las preguntas ni de la partición que se realiza en cada caso.

Por último, en las tablas VII y VIII, mostramos los resultados obtenidos sobre dos hechos que consideramos esenciales; por una parte si piensan que su respuesta se vería afectada si su nombre y su teléfono tuviera que aparecer en la encuesta (recordemos que la encuesta era anónima y que únicamente se expuso una lista de los alumnos aleatoriamente seleccionados), y por otra si estarían dispuestos a contestar, de forma veraz, a la pregunta de si es consumidor o no, sin instrumento aleatorio alguno.

De las tablas VII y VIII, podemos resaltar que el $86 \%$ de los alumnos consideran que su respuesta no se vería afectada por el hecho de que su nombre y su teléfono tuviera que aparecer en la encuesta, siendo este porcentaje ligeramente 
TABLA VII

\begin{tabular}{|c|c|c|c|c|c|c|}
\hline \multirow{2}{*}{$\begin{array}{l}\text { Nombre } \\
\text { Teléfono }\end{array}$} & \multicolumn{2}{|c|}{ Total } & \multicolumn{2}{|c|}{ Muestra 1} & \multicolumn{2}{|c|}{ Muestra 2} \\
\hline & $\mathrm{N}^{\prime \prime}$ & $\%$ & $\mathrm{~N}^{0}$ & $\%$ & $\mathrm{~N}^{\mathbf{2}}$ & $\%$ \\
\hline SI & 38 & 13,82 & 15 & 10,95 & 23 & 16,67 \\
\hline NO & 237 & 86,18 & 122 & 89,05 & 115 & 83,33 \\
\hline Total & 275 & 100,00 & 137 & 100,00 & 138 & 100,00 \\
\hline
\end{tabular}

TABLA VIII

\begin{tabular}{|c|c|c|c|c|c|c|}
\hline \multirow{2}{*}{$\begin{array}{l}\text { Pregunta } \\
\text { directa }\end{array}$} & \multicolumn{2}{|c|}{ Total } & \multicolumn{2}{|c|}{ Muestra 1} & \multicolumn{2}{|c|}{ Muestra 2} \\
\hline & $\mathrm{N}^{\prime \prime}$ & $\%$ & $\mathrm{~N}^{2}$ & $\%$ & $\mathrm{~N}^{2}$ & $\%$ \\
\hline SI & 254 & 92,36 & 127 & 92,70 & 127 & 92,03 \\
\hline NO & 21 & 7,64 & 10 & 7,30 & 11 & 7,97 \\
\hline Total & 275 & 100,00 & 137 & 100,00 & 138 & 100,00 \\
\hline
\end{tabular}

superior en la muestra 1, mientras que el $92 \%$ estarían dispuestos a manifestarnos directamente si son consumidores o no, siendo el comportamiento prácticamente idéntico en ambas muestras.

\section{ESTUDIO DE LA PROPORCION DE CONSUMIDORES}

En primer lugar se va a considerar las respuestas obtenidas al aplicar el diseño de Warner, que se recogen en la tabla IX.

Si denominamos $\pi_{A}$ a la proporción de consumidores, tenemos que aplicar las técnicas clásicas, la estimación máximo verosímil e insesgada de esta proporción

TABLA IX

\begin{tabular}{|c|c|c|c|c|c|c|}
\hline \multirow[t]{2}{*}{ Warner } & \multicolumn{2}{|c|}{ Total } & \multicolumn{2}{|c|}{ Muestra 1} & \multicolumn{2}{|c|}{ Muestra 2} \\
\hline & $\mathrm{N}^{0}$ & $\%$ & $\mathrm{~N}^{\mathrm{o}}$ & $\%$ & № & $\%$ \\
\hline SI & 89 & 32,36 & 45 & 32,85 & 44 & 31,88 \\
\hline NO & 186 & 67,64 & 92 & 67,15 & 94 & 68,12 \\
\hline Total & 275 & 100,00 & 137 & 100,00 & 138 & 100,00 \\
\hline
\end{tabular}


$\hat{\pi}_{\mathrm{A}}$, así como la de su desviación estándar, $\hat{\sigma}_{\mathrm{A}}$, es, dado que la probabilidad con que el instrumento aleatorio selecciona la pregunta íntima es de 0,7 y teniendo en cuenta tanto cada una de las muestras como el total.
Para la muestra 1: $\hat{\pi}_{\hat{A}^{\mathrm{A}}}=0,071$
Para la muestra 2: $\hat{\pi}_{A}^{A}=0,047$
$\hat{\sigma}_{A}=0,1005$
$\hat{\sigma}^{A}=0,0995$
Para el total:
$\hat{\pi}_{\mathrm{A}}^{\mathrm{A}}=0,059$
$\hat{\theta}_{A}=0,0707$

Estos resultados ponen de manifiesto la sensibilidad de este diseño frente a pequeñas variaciones en el número de respuestas afirmativas, así como la gran variabilidad de las estimaciones.

TABLA X

\begin{tabular}{lrrrr}
\hline No relacionadas & \multicolumn{2}{c}{ Muestra 1 } & \multicolumn{2}{c}{ Muestra 2 } \\
& $N^{*}$ & $\%$ & $N^{2}$ & $\%$ \\
\hline SI & 18 & 13,14 & 17 & 12,32 \\
NO & 119 & 86,86 & 121 & 87,68 \\
\hline Total & 137 & 100,00 & 138 & 100,00 \\
\hline
\end{tabular}

Considerando a continuación los resultados obtenidos al aplicar el diseño de preguntas no relacionadas, que aparecen en la tabla $X$, y teniendo en cuenta que la probabilidad con que el mecanismo de aleatorización selecciona la pregunta íntima es 0,7 en la muestra 1 y 0,6 en la muestra 2 , y suponiendo que la proporción de alumnos que acuden habitualmente en moto a la facultad, $\pi_{\mathrm{B}}$, es desconocida, podemos obtener las siguientes estimaciones máximo verosímiles e insesgadas, según las técnicas clásicas:

$$
\begin{array}{ll}
\hat{\pi}_{\mathrm{A}, \mathrm{B}}=0,156 & \hat{\sigma}_{\mathrm{A}, \mathrm{B}}=0,1431 \\
\hat{\pi}_{\mathrm{B}, \mathrm{A}}=0,074 & \hat{\sigma}_{\mathrm{B}, \mathrm{A}}=0,2623
\end{array}
$$

donde $\hat{\pi}_{\mathrm{A} . \mathrm{B}}$ y $\hat{\pi}_{\mathrm{B} . \mathrm{A}}$ son las estimaciones de $\pi_{\mathrm{A}}$ y $\pi_{\mathrm{B}}$, respectivamente, bajo el supuesto de que se desconocen estas proporciones poblacionales, y donde $\hat{\sigma}_{\mathrm{A}, \mathrm{B}} \mathrm{y}$ $\hat{\sigma}_{\mathrm{B}, \mathrm{A}}$ son las correspondientes estimaciones de $\sigma_{\mathrm{A}}$ y $\sigma_{\mathrm{B}}$, respectivamente, bajo el mismo supuesto.

La proporción de consumidores supera en mucho a la obtenida mediante el diseño de Warner, aunque el valor tan elevado de la desviación estándar puede 
poner en duda la validez de esta estimación. Ahora bien, es evidente que se pued obtener información sobre la proporción de alumnos que acuden habitualment en moto al Centro. Así, se contaron durante los ocho días en que se realizaron la encuestas el número de motos que se encontraban en el aparcamiento de 1 Facultad, tanto en el turno de la mañana como en el de la tarde, y suponiendo qu cada moto transporta a un alumno y medio, y a partir del número medio de esto vehículos se ha estimado esta proporción, que ha alcanzado un valor de 0,077 De esta forma, y aplicando nuevamente las técnicas clásicas, podemos obtene las siguientes estimaciones máximo verosímiles e insesgadas para cada una de la. muestras:

Para la muestra 1: $\hat{\pi}_{\mathrm{A} / \mathrm{B}}=0,155$

$$
\begin{aligned}
& \hat{\sigma}_{\mathrm{A} / \mathrm{B}}=0,0412 \\
& \hat{\sigma}_{\mathrm{A} / \mathrm{B}}=0,0469
\end{aligned}
$$

donde $\hat{\pi}_{\mathrm{A} / \mathrm{B}}$ y $\hat{\sigma}_{\mathrm{B} / \mathrm{A}}$ son las correspondientes estimaciones de $\pi_{\mathrm{A}}$ y $\sigma_{\Lambda}$, bajo e supuesto de que $\pi_{\mathrm{B}}$ es conocida.

Podemos observar que la proporción de consumidores sigue situándose er tomo al 15\%, mientras que la desviación estándar ha disminuído notablemente.

Ahora bien, afirmar que la proporción de alumnos que acuden en moto a le Facultad es 0,077 parece, en principio, muy arriesgado. También debemos tene1 en cuenta que el trabajo "Los andaluces ante las drogas" (Junta de Andalucía. 1989), estima en un $12,9 \%$ la proporción de individuos que, con estudios mediossuperiores, han consumido alguna vez durante los últimos seis meses cánnabis.

Por ello, y dado que las distribuciones beta parecen ser razonables aproximaciones para una extensa variedad de tipos de información a priori sobre la proporción de la población que posee cierta característica, proponemos como distribución inicial del parámetro $\pi_{A}$, la distribución beta $B\left(a_{1}, b_{1}\right)$, y como distribución inicial del parámetro $\pi_{B}$, la distribución beta $B a_{2}, b_{2}$ ). Así, si suponemos que la moda de $\pi_{\mathrm{A}}$ es 0,12 y su desviación estándar la ciframos en 0,04 , podemos determinar, aproximadamente, los valores de $a_{1}$ y $b_{1}$, resolviendo el sistema:

$$
\begin{aligned}
& 0,12=\left(a_{1}-1\right) /\left(a_{1}+b_{1}-2\right) \\
& 1 / 0,04^{2}=-\left[\frac{\partial 2}{\partial \pi^{2}{ }_{A}} \ln f\left(\pi_{A} / a_{1}, b_{1}\right)\right] \pi_{A}=0,12
\end{aligned}
$$

siendo entonces $a_{\mathrm{T}}=8,92$ y $\mathrm{b}_{1}=59,08$. Análogamente, considerando que la moda de $\pi_{\mathrm{B}}$ es 0,077 y su desviación estándar 0,05 ; tenemos que $\mathrm{a}_{2}=3,19$ y $\mathrm{b}_{2}=27,24$. 
De esta forma, la moda de la distribución final de la proporción de individuos que tienen la consideración de consumidores, $\pi_{\Lambda}$, utilizando el algoritmo EM, la desviación estándar de esta distribución final, $\sigma_{\mathrm{A}}$, que se calcula a partir de las aproximaciones de los dos primeros momentos de la distribución final (Tierney y Kdane, 1986), así como la mejor aproximación a la distribución final, son:

Diseño de Warner:

$\begin{array}{lll}\text { Muestra 1: } & \hat{\pi}_{A}=0,114 & \hat{\sigma}_{A}=0,0359 \mathrm{~B}(9,99 ; 71,08) \\ \text { Muestra 2: } & \hat{\pi}_{\mathrm{A}}=0,111 & \hat{\sigma}_{\mathrm{A}}=0,0354 \mathrm{~B}(9,89 ; 72,46) \\ \text { Total: } & \hat{\pi}_{\mathrm{A}}=0,106 & \hat{\sigma}_{\mathrm{A}}=0,0323 \mathrm{~B}(11,15 ; 86,08)\end{array}$

Diseño de preguntas no relacionadas:

$\begin{array}{lll}\text { Muestra 1: } & \hat{\pi}_{A}=0,136 & \hat{\sigma}_{A}=0,0337 \mathrm{~N}(0,136 ; 0,033) \\ \text { Muestra 2: } & \hat{\pi}_{\mathrm{A}}=0,132 & \hat{\sigma}_{\mathrm{A}}=0,0361 \mathrm{~N}(0,132 ; 0,035)\end{array}$

donde $N(\mu ; \sigma)$ representa una distribución normal de media $\mu$ y desviación estándar $\sigma$.

Podemos observar que al utilizar las técnicas bayesianas, bajo el supuesto que las distribuciones iniciales de los parámetros son correctas, las estimaciones de las desviaciones estándares obtenidas alcanzan unos valores que podemos considerar adecuados, aunque la diferencia de las modas estimadas según el diseño empleado se sigue poniendo de manifiesto, aunque para el de Warner, estas diferencias se han recortado notablemente. Así pues, y puesto que mediante el diseño de preguntas no relacionadas se ha estimado que la proporción de alumnos que acuden habitualmente en moto a la facultad, utilizando las técnicas clásicas, prácticamente es igual a nuestra estimación basada en el número de motos aparcadas, nos inclinamos a pensar que este diseño ofrece mejores resultados.

También, y puesto que la información utilizada para estimar la distribución inicial de la proporción de consumidores, se obtuvo en el año 1987, y además no se ajusta exactamente a nuestra encuesta, puesto que nuestros alumnos todavía están estudiando y que la consideración de consumidor está referida a los últimos treinta días y no a los seis meses anteriores, preferimos considerar como distribución inicial de este parámetro una beta $\mathrm{B}(1 ; 1)$, aunque se incremente la estimación de la desviación estándar. De esta forma, bajo este último supuesto tenemos:

Muestra 1: $\hat{\pi}_{\mathrm{A}}=0,155$
Muestra 2: $\hat{\pi}_{\mathrm{A}}=0,154$

$$
\begin{aligned}
& \hat{\sigma}_{A}=0,0442 \mathrm{~N}(0,155 ; 0,045) \\
& \hat{\sigma}_{\mathrm{A}}=0,0539 \mathrm{~N}(0,154 ; 0,055)
\end{aligned}
$$




\section{CONCLUSIONES DE LA ENCUESTA}

La experimentación que hemos llevado a cabo en la Facultad de Ciencia Económicas y Empresariales de la Universidad de Sevilla ha perseguido funda mentalmente valorar la actitud de los entrevistados cuando se les realiza un encuesta que incorpora un instrumento aleatorio, y además aplicar los desarrollo teóricos que hasta la fecha hemos desarrollado en lo concerniente a la aplicació] del algoritmo EM y las aproximaciones a la distribución final cuando se utiliza técnicas bayesianas.

Así pues, debemos mencionar en primer lugar que nuestros alumnos conside ran que su intimidad queda adecuadamente protegida al utilizar alguno de los do diseños propuestos, sin que existan diferencias notables entre ellos. Ahora bien este colectivo alcanza un cierto nivel cultural, y eso en principio, tal y comı afirman algunos autores, favorece notablemente este aspecto. Sin embargo, cree mos que la utilización de un instrumento aleatorio tan fácilmente comprensibl como las cartas, supondría, sin importar sobre manera el nivel cultural del entre vistado, su comprensión hacia el hecho de que es imposible que el entrevistado conozca la pregunta que le ha correspondido. También resaltar que los alumno han manifestado que consideran más claro el diseño de Warner que el de pregun tas no relacionada, aunque tal y como hemos mencionado creemos que est: situación se debe a que no han comprendido este último diseño, puesto que nı encontraban relación alguna entre ambas características, aunque, evidentemente la hipótesis de independencia entre ellas es esencial para el desarrollo del diseño Por último, mencionar que, en principio, los entrevistados no se muestran contra rios a que su nombre y teléfono aparezca en la encuesta, y que parecen dispuest a contestarnos directamente si son consumidores, en los términos en los que se hi definido.

En segundo lugar, y a la vista de los resultados obtenidos en la estimació tanto de la proporción de consumidores como de su desviación estándar, la incor poración de las técnicas bayesianas, cuando la información inicial sobre uno, c ambos, de los parámetros es adecuada, reduce notablemente la sensibilidad qu ha presentado el diseño de Warner hacia el número de respuestas afirmativas, ! logra, en ambos diseños, una mayor eficiencia, medida en términos de la caracte rística no relacionada, o sea, la proporción de alumnos que acuden habitualment en moto a la Facultad, ha sido fundamental para considerar más adecuados lo: resultados obtenidos por el diseño de preguntas no relacionadas que por el df Warner. Por último debemos resaltar que al emplear las técnicas bayesianas en e diseño de preguntas no relacionadas, se reducen notablemente los costes de análisis, ya que únicamente hace falta una muestra, situación que, cuando st 
utilizan las técnicas clásicas, sólo se puede presentar si se conoce exactamente la proporción de individuos que poseen la característica no relacionada, y que no podemos considerar como general para la mayoría de los estudios.

\section{CONSIDERACIONES FINALES}

La sociedad actual presenta tres grandes problemas, como son el terrorismo, el paro y la droga. Precisamente sobre el consumo de drogas debemos realizar una breve reflexión, puesto que si no disponemos de datos sobre el consumo, no se podrá realizar una adecuada prevención, ya que la información es el punto de partida imprescindible para su planeamiento.

En esta línea, es el propio Comisionado para la droga de la Consejería de Salud y Servicios Sociales de la Junta de Andalucía quien afirma que estos datos no se toman, a veces, con la suficiente fiabilidad (Los andaluces ante las drogas, 1989). Y es precisamente en este punto donde las técnicas estadísticas deben ser aplicadas para lograr una mayor fiabilidad en la información recogida mediante una muestra. Entonces, aunque el diseño de la misma sea correcto, no se suelen considerar aquellos errores que son ajenas al muestreo, esto es, las respuestas delibedaramente falsas y la propia falta de respuesta.

De esta forma, al considerar aquellas drogas que son ilegales, o que están consideradas socialmente indeseables, se debe facilitar al entrevistado de un mecanismo que protega su intimidad, con lo que se estará contribuyendo a facilitar su cooperación, logrando así reducir dichos errores.

Ahora bien, debemos resaltar que los diseños de respuesta aleatorizada incrementan los costes de una encuesta, tanto por la vía de aumentar el tamaño muestral necesario para conseguir la misma eficiencia en los estimadores, como por la de incrementar el tiempo para que el entrevistador realice la encuesta, puesto que es imprescindible que al entrevistado se le presente el mecanismo de aleatorización y que compruebe que su intimidad queda adecuadamente protegida.

Por otro lado, también se tienen que considerar las limitaciones que presentan los diseños de respuesta aleatorizada, pues para estimar los valores de una variable policotómica o de una variable contínua, la aplicación de éstos es, normalmente, compleja.

A pesar de todo ello, creemos que la utilización de estos diseños conduciría a obtener resultados más fiables y precisos que los conseguidos mediante la aplicación de las técnicas clásicas, siempre y cuando, se pretenda obtener información sobre aquellas drogas que bien estén mal consideradas socialmente o bien sean ilegales. 


\section{BIBLIOGRAFIA}

Antes de ofrecer las referencias bibliográficas, queremos dejar constancia de nuestro agradecimient a Susana León Sánchez y a Vicenta María Márquez de la Plata y Cuevas, por su inestimable colaboració en el diseño, tabulación y análisis de la encuesta.

ABUL-ELA, A. A., GREENBERG, B. G. and HORVITZ, D. G. (1967). A multiproportion randomizer response model. J. Amer. Statist. Assoc. 62, 990-1008.

ARIAS, C. (1990). Análisis bayesiano de los diseños de respuesta aleatorizada. Tesis Doctoral. Universi dad de Sevilla. Pendiente de publicación.

BOURKE, P.D. (1974) Multi-proportions randomized responde using the unrelated question technique Report 74, Errors in Surveys Research Project. Inst. of Satist., University of Stockholm.

BOURKE, P.D. (1974) Multi-proportions randomized responde using the unrelated question technique Report 68, Errors in Surveys Research Project. Inst. of Satist., University of Stockholm.

BOURKE, P.D. and MORAN M.A. (1988). Estimating proportions from randomized response data using the EM algorithm. J. Amer. Statist. Assoc. 83, 964-968.

DEMPSTER, A. P. LAIRD, N. M. and RUBIN, D. B. (1977). Maximum likelihood estimation from incomplete data via the EM algorithm (with discussion). J. Roy. Statist. Soc. B 39, 1-38.

DIEZ DE CASTRO, E. MARTIN, E., SERRANO F. y BASULTO, J. (1988). Informe sobre actitudes opiniones e imagen de la Universidad de Sevilla. Universidad de Sevilla.

ERIKSSON, S.A. (1973). A new model for randomized response. Internat. Statist. Rev., 41, 101-113.

FOLSOM, R. E., GREENBERG, B. G., HORVITZ, D. G. and ABERNATHY, J.R. (1973). The two alternate questions randomized response model for human surveys. J. Amer. Satist. Assoc., 68, 525 530.

GREENBERG, B. G., ABDEL-LATIF, A., ABUL-ELA, A. A., SIMMONS, W. R. and HORVITZ, D.G (1969). The unrelated question randomized response model: theoretical framework. J. Amer. Statist Assoc. 64, 520.539 .

HARTLEY, H. O.(1958). Maximun likelihood estimation from incomplete data. Biometrics, 14, 174 194.

HORVITZ, D. G. SHAH, B. V. and SIMMONS, W. R. (1967). The unrelated question randomizec response model. Proc. ASA. Soc. Statist. Sec., 65-72.

\section{ANEXO}

\section{Cuestionario nำ 1}

Fecha:

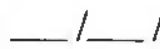

1. Edad:

2. Sexo:

1 Hombre

2 Mujer

3. Supuesto que tiene la consideración de CONSUMIDOR todo aquel que haya consumido alguna vez, durante los últimos 30 días, Cánnabis, esto es. Haschis, Marihuana, Porros, póngase donde el entrevistador no pueda verle, baraje las cartas y extraiga una al azar, que sólo debe ser vista por usted. Conteste SI o NO a la declaración que aparece impresa en la carta extraída. 


\section{RESPUESTA:}

Introduzca la carta en la baraja, y barájelas para que nadie sepa la carta que usted ha extraído.

4. ¿Cree usted que su intimidad queda adecuadamente protegida al utilizar la baraja en lugar de preguntarle directamente? 1 SI 2 NO

5. El entrevistador va a cambiarle la baraja. Bajo el supuesto referido en la cuestión cuarta, conteste SI o NO a la declaración que aparece impresa en la carta extraída.

\section{RESPUESTA:}

Introduzca la carta en la baraja, y barájelas para que nadie sepa la carta que usted ha extraído.

6. ¿Cree usted que su intimidad queda adecuadamente protegida al utilizar la baraja en lugar de preguntarle directamente?

\section{SI 2 NO}

7. ¿Cuál de los dos métodos propuestos cree que es más claro?

$1 \mathrm{El}$ primero $2 \mathrm{El}$ segundo 3 Ninguno 4 Los dos por igual

8. ¿Cuál de los dos métodos propuestos cree que protege más su intimidad?

1 El primero 2 El segundo 3 Ninguno 4 Los dos por igual

9. Si su nombre y su teléfono tuviera que aparecer en el cuestionario, ¿Cree que se vería afectada la respuesta?

$1 \mathrm{SI}$

$2 \mathrm{NO}$

10. Si se le preguntara si usted es consumidor o no lo es, ¿Estaría dispuesto a contestar, de forma veraz, a la pregunta?

$1 \mathrm{SI} 2 \mathrm{NO}$ 